\title{
Laboratory-Generated Comment
}

National Cancer Institute

\section{Source}

National Cancer Institute. Laboratory-Generated Comment. NCI Thesaurus. Code C70932.

Additional information related to the specific test that was generated in the laboratory where test had been performed. This comment is usually provided in free text format. 